\title{
Skp2 is a promising therapeutic target in breast cancer
}

\section{Zhiwei Wang ${ }^{1}$, Hidefumi Fukushima ${ }^{1}$, Hiroyuki Inuzuka ${ }^{1}$, Lixin Wan ${ }^{1}$, Pengda Liu ${ }^{1}$, Daming Gao ${ }^{1}$, Fazlul H. Sarkar ${ }^{2}$ and Wenyi Wei ${ }^{1}{ }^{*}$}

${ }^{1}$ Department of Pathology, Beth Israel Deaconess Medical Center, Harvard Medical School, Boston, MA, USA

${ }^{2}$ Department of Pathology, Karmanos Cancer Institute, School of Medicine, Wayne State University, Detroit, MI, USA

\section{Edited by:}

Hui-Kuan Lin, The University of Texas MD Anderson Cancer Center, USA

\section{Reviewed by:}

Jianping Jin, The University of Texas Health Science Center at Houston, USA

Ingrid E. Wertz, Genentech Inc., USA

\section{${ }^{*}$ Correspondence:}

Wenyi Wei, Department of Pathology, Beth Israel Deaconess Medical

Center, Harvard Medical School, 330

Brookline Avenue, Boston, MA

02215, USA.

e-mail:wwei2@bidmc.harvard.edu
Breast cancer is the most common type of cancer among American women, and remains the second leading cause of cancer-related death for female in the United States. It has been known that several signaling pathways and various factors play critical roles in the development and progression of breast cancer, such as estrogen receptor, Notch, PTEN, human epidermal growth factor receptor 2, PI3K/Akt, BRCA1, and BRCA2. Emerging evidence has shown that the F-box protein S-phase kinase associated protein 2 (Skp2) also plays an important role in the pathogenesis of breast cancer. Therefore, in this brief review, we summarize the novel functions of Skp2 in the pathogenesis of breast cancer. Moreover, we provide further evidence regarding the state of our knowledge toward the development of novel Skp2 inhibitors especially natural "chemopreventive agents" as targeted approach for the prevention and/or treatment of breast cancer.

Keywords: Skp2, breast cancer, therapy, signaling pathway, ubiquitination, cell cycle

\section{INTRODUCTION}

Breast cancer is the most common female malignancy, and the second leading cause of cancer-related death after lung cancer in the United States (Siegel et al., 2011). The American Cancer Society estimates that approximately 230,480 American women will be diagnosed with breast cancer and almost 40,000 will die from it in 2011 (Siegel et al., 2011). Breast cancer has been clinically characterized by the expression of hormone and growth factor receptors such as estrogen receptor (ER), progesterone receptor (PR), and human epidermal growth factor receptor 2 (Her2; Lin et al., 2010). Approximately $70 \%$ of breast cancer patients have elevated expression of ER and PR, while $20 \%$ of breast tumors have overexpression of Her2. The remaining $10 \%$ of breast cancer cases are classified as triple-negative breast cancers due to the lack of expression of ER, PR, and Her2 (Lin et al., 2010). Even though breast cancer recurrence rates have been significantly decreased due to early detection and adjuvant therapies, breast cancer still causes high mortality. Current therapies for breast cancer include surgery, radiation, chemotherapy, hormonal, and biological therapies (Lin et al., 2010). Despite improved treatments that have been achieved recently, many breast tumors are not eradicated effectively due to their intrinsic or acquired resistance, or relapse following initial response, resulting in metastatic disease at later stages that leads to patient death (Lin et al., 2010). Therefore, this disappointing outcome suggests that further understanding of the molecular mechanisms underlying the development of breast cancer, especially the critical events of the metastatic spread, is essential to identify new therapeutic targets for achieving better treatment of breast cancer.

In recent years, it has been demonstrated that multiple cellular signaling pathways including the ER, PR, epidermal growth factor receptor (EGFR), breast cancer susceptibility gene (BRCA), PI3K (phosphatidylinositol 3-kinase), PTEN (phosphatase and tensin homolog on chromosome 10), mammalian target of rapamycin
(mTOR), MAPK (mitogen-activated protein kinases), androgen receptor (AR), Akt, nuclear factor- $\kappa \mathrm{B}(\mathrm{NF}-\kappa \mathrm{B})$, receptor of activated nuclear factor kappa ligand (RANKL), Wnt, sonic hedgehog (SHH), platelet-derived growth factor (PDGF), and Notch pathways have been found to play important roles in the development and progression of breast cancer (Foley et al., 2010; Haagenson and Wu, 2010; Nahta and O'Regan, 2010; Narod, 2010; Prosperi and Goss, 2010; Rosen et al., 2010; Visbal and Lewis, 2010; Guo et al., 2011; Hernandez-Aya and Gonzalez-Angulo, 2011; O'Regan and Hawk, 2011). It is worth noting that exact mechanisms by which breast cancer arises remain largely unclear. Emerging evidence has demonstrated that S-phase kinase associated protein 2 (Skp2) is critically involved in the pathogenesis of breast cancer (Hulit et al., 2006; Sun et al., 2007; Fujita et al., 2008; Voduc et al., 2008; Chan et al., 2010b). Therefore, in this review article, we will provide an overview on the role of Skp2 oncoprotein, and summarizing recently published literatures that highlight the novel roles of Skp2 in mammary tumorigenesis. Finally, we will also summarize approaches to inhibit Skp2 and would suggest that Skp2 could be a promising therapeutic target in combating human breast cancer.

\section{Skp2 IS ONE OF THE COMPONENTS OF THE SCF E3 LIGASE COMPLEX}

S-phase kinase associated protein 2 belongs to ubiquitinproteasome system (UPS) that plays vital roles in regulating many biological processes by controlling the timely turn-over of proteins (Frescas and Pagano, 2008). UPS includes three types of enzymes for exerting UPS function via a cascade of enzymatic reactions (Nalepa et al., 2006). The three enzymes are ubiquitin-activating enzyme (E1), ubiquitin-conjugating enzyme (UBC, also known as E2), and ubiquitin ligase (E3; Weissman et al., 2011). The initial step is to add ubiquitin to E1 and subsequently transfer of it to the E2 enzyme. The ubiquitin-charged E2 then interacts with E3 and 
transfers the ubiquitin onto the substrate, leading to the formation of a mono-ubiquitin or polyubiquitin chain due to the consecutive addition of ubiquitin moieties to target proteins (Nakayama and Nakayama, 2006). It is evident that the specificity of substrate selection depends on the E3, leading to substrate degradation by the 26S proteasome (Nakayama and Nakayama, 2006). This biochemical feature could explain why deregulation of E3 has been frequently found in carcinogenesis, whereas the function of E1 and E2 in carcinogenesis documented only few published reports (Okamoto et al., 2003).

It is known that Skp2 is one of the components of the Skp1Cullin1-F-box (SCF) E3 ligase complex. The SCF complexes comprise a family of multi-subunit E3 ubiquitin ligases that target selected proteins for destruction by the $26 \mathrm{~S}$ proteasome degradation (Frescas and Pagano, 2008). SCF complexes contain several constant core proteins such as Skp1, Rbx1 (also known as ROC1), Cullin1, and a variable subunit called F-box protein (Frescas and Pagano, 2008). F-box protein that functions as a receptor for target proteins can bind to a specific subset of substrates for promoting their degradation (Nakayama and Nakayama, 2006). So far, more than 70 putative F-box proteins encoded in the human genome have been found (Nakayama and Nakayama, 2006). It is clear that Skp2 is an F-box protein of the SCF complex (Figure 1). Because Skp2 is originally discovered with Skp1 associated with the S-phase kinase Cdk2/cyclin A, it was named as Skp2 (Frescas and Pagano, 2008). Among the many F-box proteins, Skp2 has been well-characterized and it has been shown to be involved in carcinogenesis.

\section{Skp2 IS A PROT0-ONCOPROTEIN}

$\mathrm{S}$-phase kinase associated protein 2 is the specific factor of the $\mathrm{SCF}^{\text {skp2 }}$ E3 ligase involved in cell cycle progression through degradation of its targets (Bashir and Pagano, 2004; Frescas and Pagano, 2008). For example, Skp2 is essential for p27 degradation and thereby limiting cells in G1 phase, prior to entry into S-phase (Amati and Vlach, 1999; Carrano et al., 1999; Tsvetkov et al., 1999). This finding raised the question of whether the observed lower

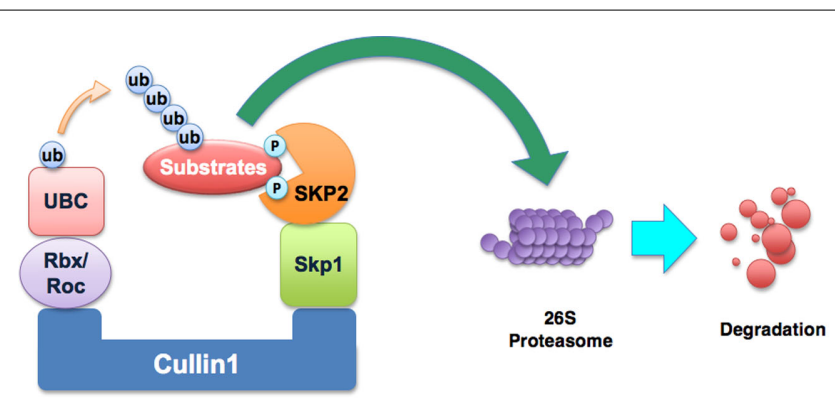

FIGURE 1 | Illustration of the SCFskp2 complex. The SCF

(Skp1-Cullin1-F-box) E3 ubiquitin ligase complex consists of four components: Skp1, Rbx1, Cullin1, and the variable F-box protein. In SCFskp2, the F-box protein component is Skp2. Skp2 recognizes the targeted proteins and makes the ubiquitin transfer to the substrate protein by UBC (the E2 enzyme). The addition of polyubiquitin targets substrates for degradation by the $26 \mathrm{~S}$ proteasome. The known substrates of Skp2 include p21, p27, p57, p130, Tob1, FOX01, and c-Myc. levels of p27 are caused by overexpression of Skp2 in human cancers. Indeed, accumulating evidence has shown that Skp2 expression is inversely correlated with p27 levels in different types of human malignancies (Hershko and Shapira, 2006). To date, in addition to $\mathrm{p} 27$, specific substrates of Skp2 have also been identified which include p57 (Kamura et al., 2003), p21 (Yu et al., 1998), p130 (Tedesco et al., 2002; Bhattacharya et al., 2003), Tob1 (Hiramatsu et al., 2006), FOXO1 (Huang et al., 2005), and many others (Michel and Xiong, 1998; Yu et al., 1998; Nakayama et al., 2000). These substrates are involved in many cellular processes such as cell cycle regulation, proliferation, differentiation, apoptosis, and survival. Without a doubt, Skp2 has important functions in the regulation of these cellular processes due to degradation of its substrates, most of which are tumor suppressor proteins (Gstaiger et al., 2001). Because Skp2 is responsible for the degradation of the above-mentioned tumor suppressor proteins, Skp2 is thought to function as oncoprotein (Gstaiger et al., 2001).

Many studies have shown that overexpression of Skp2 is observed in a variety of human cancers, including lymphomas (Seki et al., 2003), prostate cancer (Wang et al., 2011a), colorectal cancer (Li et al., 2004), melanoma (Rose et al., 2011), nasopharyngeal carcinoma (Fang et al., 2009; Xu et al., 2011), pancreatic cancer (Schuler et al., 2011), and breast carcinomas (Radke et al., 2005; Zheng et al., 2005). Moreover, Skp2 has been found to be a prognostic marker in multiple cancers. For example, overexpression of Skp2 is associated with late metastases to lymph nodes leading to poor survival in colorectal cancer (Li et al., 2004). A separate study showed that overexpression of Skp2 was associated with significantly poorer tumor differentiation and reduced patient survival in gastric cancer (Ma et al., 2005). Similarly, Skp2 expression was correlated with histological grade and tumor size in hepatocellular carcinoma (Lu et al., 2009). Moreover, elevated Skp2 expression is related with tumor metastasis in melanoma (Rose et al., 2011), lymphoma, oral squamous cell carcinomas (Tosco et al., 2011), pancreatic cancer (Einama et al., 2006), and prostate cancer (Wang et al., 2011a). Furthermore, Skp2 expression has been considered as a biomarker for poor prognosis in breast cancer (Voduc et al., 2008), melanoma (Rose et al., 2011), and nasopharyngeal carcinoma (Xu et al., 2011). Interestingly, one recent report showed that Skp2 mediates resistance of pancreatic cancer cell lines toward the TRAIL (tumor factor-related apoptosis-inducing ligand)-induced apoptosis (Schuler et al., 2011). Taken together, Skp2 is believed to be a proto-oncoprotein. In the following paragraphs, we will discuss the role of Skp2 in the breast cancer progression.

\section{THE ROLE OF SKP2 IN DEVELOPMENT AND PROGRESSION OF BREAST CANCER}

A growing body of literature strongly suggests that Skp2 plays critical roles in the breast tumorigenesis. For example, Skp 2 has been reported to be overexpressed in a subset of breast carcinomas with low level of p27 expression (Signoretti et al., 2002). Emerging evidence also reveals that Skp2 plays important roles in cell growth, apoptotic cell death, invasion, and metastasis in human breast cancer (Zheng et al., 2005; Sonoda et al., 2006). Multiple signaling pathways, such as phosphatidylinositol 3-kinase (PI3K)/Akt (Gao et al., 2009a), ERK (extracellular signal-regulated kinase; Lin and Yang, 2006), peroxisome proliferator-activated receptor- $\gamma$ 
(PPAR $\gamma$; Zaytseva et al., 2008), IGF-1 (insulin-like growth factor1; Lu et al., 2004), and mTOR (Shapira et al., 2006) signaling have been discovered to cross-talk with Skp2, and thus it is believed that the cross-talks between Skp2 and these signaling pathways may play important roles in breast cancer. However, the molecular mechanism(s) by which Skp2 facilitates breast cancer remain largely elusive. Here, we will discuss some recent advances in the understanding on the role of Skp2 in breast tumor progression. Therefore, in the following sections, we will summarize the results of emerging studies on the Skp2 as well as its therapeutic implication in human breast cancer.

\section{Skp2 IS OVEREXPRESSED IN BREAST CANCER}

It has been widely accepted that Skp2 is frequently overexpressed in a variety of human cancers including breast cancer. For instance, both Skp2 mRNA and protein display elevated levels in breast cancer cell lines and primary breast tumors (Radke et al., 2005; Fujita et al., 2008). Specifically, using microarray analysis and immunohistochemistry, Pagano's group found that higher levels of Skp2 are present more frequently in ER-negative tumors than in the ER-positive cases (Signoretti et al., 2002). Moreover, Skp2B, an isoform of Skp2, is also overexpressed in breast cancer cell lines and primary breast tumors (Radke et al., 2005). Since Skp2 is overexpressed in human breast cancer, inhibition of Skp2 could be a promising therapeutic strategy for breast cancer treatment.

\section{Skp2 PROMOTES CELL GROWTH IN BREAST CANCER}

Emerging evidence is accumulating showing that Skp2 promotes cancer cell growth including breast cancer cell growth. For example, down-regulation of Skp2 by RNA interference significantly inhibited cell proliferation in MCF-7 breast cancer cells (Sun et al., 2007). Treatment with Skp2 siRNA followed by treatment with epirubicin further inhibited the proliferation of breast cancer cell lines (Sun et al., 2007). Consistent with the role of Skp2 in cell growth, Signoretti et al. (2002) found that inhibition of Skp2 induced a decrease of adhesion-independent growth in both ER-positive and ER-negative breast cancer cells. A study from Wan's group has also shown that overexpression of Skp2 enhanced cell proliferation in normal breast cell line MCF10A, while depletion of Skp2 reduces cellular growth in breast cancer cell line (Fujita et al., 2008). Lee and McCormick (2005) also found similar results, which showed that down-regulation of Skp2 inhibits the in vitro growth of glioblastoma cells. Moreover, knockdown of endogenous Skp2 by siRNA treatment also inhibited the in vivo tumor growth in nude mice (Sun et al., 2007). Furthermore, xenograft expressing high levels of Skp2B grows faster than xenograft expressing low levels of Skp2B (Radke et al., 2005), suggesting that Skp2B could also promote breast tumor growth. Recently, it has been found that Skp2B interacts with the REA (repressor of estrogen receptor activity) and that overexpression of Skp2B leads to a decreased REA levels, suggesting that Skp2B contributes to breast cancer in part by modulating the activity of the ER (Umanskaya et al., 2007). More recently, Chander et al. (2010) demonstrated that Skp2B attenuates the p53 activity by degradation of prohibitin, suggesting that his effect is independent of p300 in breast cancer.

\section{Skp2 INHIBITS CELL APOPTOSIS IN BREAST CANCER}

Inhibition of cell growth by down-regulation of Skp2 raised one question of whether the observed cell growth inhibition is caused by a possible increase in cellular apoptosis. Multiple studies have addressed this question. Indeed, Skp2 has been found to be involved in regulating cellular apoptosis in various types of human cancer cells (Kitagawa et al., 2008). Kitagawa et al. (2008) demonstrated that reducing the expression of Skp2 increased DNA-damage-mediated apoptosis in multiple cancer cells, while overexpression of Skp2 suppressed p53-mediated apoptosis. The reason is that Skp2 suppressed p300-mediated acetylation of p53 and subsequent transactivation ability of p53 through forming a complex with p300 (Kitagawa et al., 2008). It has also been reported that down-regulation of Skp2 caused apoptosis via induction of p27 in glioblastoma cells (Lee and McCormick, 2005). Moreover, down-regulation of both Skp2 and p27 increased apoptosis synergistically (Lee and McCormick, 2005). Huang et al. (2005) also found that overexpression of Skp2 inhibits transactivation of FOXO1 and abolishes the induced effect of FOXO1 on cell apoptosis in prostate cancer. Similar trends were found in breast cancer cell lines. For example, in breast cancer MCF-7 cells, knockdown of Skp2 by RNAi increased cellular apoptosis (Sun et al., 2007). Taken together, these results indicate that Skp2 could inhibit cell apoptosis in breast cancer cells. However, more thorough studies are required to fully understand the underlying molecular and signaling events by which Skp2 influences the cellular apoptotic decision.

\section{Skp2 REGULATES CELL CYCLE IN BREAST CANCER}

The cell cycle is tightly controlled by multiple regulatory mechanisms to ensure ordered and coordinated cell cycle progression. It is known that a major mechanism to ensure the orchestrated cell cycle is to degrade key regulators governing cell cycle progression by the UPS. SCF has been considered to be a major driving force controlling proper cell cycle progression through ubiquitination of G1 cyclins and CDK inhibitors (Skaar and Pagano, 2009). For example, Skp2 targets numerous substrates for degradation, many of which are negative cell cycle regulators such as p27 (Carrano et al., 1999; Tsvetkov et al., 1999), p57 (Kamura et al., 2003), p21 (Yu et al., 1998), p130 (Tedesco et al., 2002; Bhattacharya et al., 2003), and FOXO1 (Huang et al., 2005). Therefore, Skp2 has been found to correlate with dysregulation of cell cycle in human cancers including breast cancer. We have discovered that Akt regulates cell cycle through modulating Skp2 activity and its destruction by APC/Cdh1 (Gao et al., 2009a,b). Fujita et al. (2008) found that overexpression of Skp2 in MCF10A breast epithelial cells significantly elevated the fraction of cells in S-phase, suggesting that an increase in Skp2 protein levels could lead to an aberrant cell cycle. Consistent with these findings, the fraction of cells in S-phase was significantly reduced in Skp2depleted MCF-7 breast cancer cells (Fujita et al., 2008). Moreover, Skp2 expression abrogates antiestrogen-mediated cell cycle arrest in hormone-dependent breast epithelial cancer cells (Signoretti et al., 2002). Further research toward exploration of the molecular mechanisms by which Skp2 regulates cell cycle requires in-depth investigations. 


\section{Skp2 PROMOTES TUMOR METASTASIS IN BREAST TUMOR}

S-phase kinase associated protein 2 overexpression has been correlated with tumor progression such as stage and recurrence in human cancers (Einama et al., 2006), indicating that Skp2 may be important in cancer cell migration, invasion, and metastasis. Many studies have shown the positive relationship between Skp2 expression and tumor metastasis in human cancers (Tosco et al., 2011). For example, Tosco et al. (2011) found the correlation between Skp2 expression and nodal metastasis in oral squamous cell carcinomas. Hung et al. (2010) reported that Skp2 overexpression increased the expression of MMP-2 and MMP-9, leading to cell invasion in lung cancer cells. Consistent with this notion, Einama et al. (2006) also found that higher level Skp2 expression was correlated with the extent of lymph node metastasis in pancreatic ductal adenocarcinoma. Moreover, overexpression of Skp2 was detected more frequently in tumors metastatic to the axillary lymph nodes in breast cancer (Zheng et al., 2005), indicating that Skp2 could promote breast tumor metastasis. Recently, Lin's group identified one mechanism by which Skp2 increases tumor metastasis. Their elegant work demonstrated that Skp2 cooperates with Myc to induce RhoA transcription via recruiting Miz1 and p300 to the RhoA promoter (Chan et al., 2010a). Since RhoA plays a crucial role in cancer metastasis, deficiency of Skp2-Myc-Miz1p300 transcriptional complex led to impaired RhoA expression, resulting in the inhibition of cell migration, invasion, and subsequent breast cancer metastasis (Chan et al., 2010a). Moreover, deficiency of Skp2 profoundly restricted breast cancer metastasis to the lung, whereas overexpression of Skp2 promoted the metastatic events (Chan et al., 2010a), suggesting a critical role for Skp2 in promoting breast cancer metastasis.

\section{Skp2 PREDICTS FOR POOR PROGNOSIS IN BREAST CANCER}

It has been reported that there is a statistically significant association between Skp2 expression levels and breast tumor grades. Moreover, high expression levels of Skp2 are associated with poor survival (Signoretti et al., 2002). Furthermore, Voduc et al. (2008) found that the combination of Skp2 and high cyclin E expression predicts poor prognosis in breast cancer and it is associated with high risk features. Davidovich et al. (2008) also demonstrated that Skp2 expression was inversely related to p27 levels, tumor grade, as well as expression of ER and PR. Both Skp2 and p27 were suggested to be accurate prognostic markers for disease-free and overall survival in breast cancer. Interestingly, one study showed that low p27 level and high Skp2 level were not associated with disease-free survival in breast cancer, although low p27 and high Skp2 were related to unfavorable prognostic factors including larger tumor size, higher grade tumor, ER- and PR-negative, and Her2 overexpression (Ravaioli et al., 2008). Taken together, it is obvious that further in-depth investigations are needed to confirm whether Skp2 could be a promising prognostic marker for breast cancer patients or not.

\section{Skp2 IS INVOLVED IN DRUG RESISTANCE IN BREAST CANCER}

Chemotherapy is the most important treatment strategy for human cancers including breast cancer. However, chemotherapy often fails to cure cancer due to the acquisition of drug resistance phenotype of cancer cells (Wang et al., 2010b). Thus, increasing drug sensitivity could offer better treatment for human cancer patients (Wang et al., 2011b). Recently, several studies have shown that Skp2 is involved in drug resistance in human cancers including breast cancer (Ishii et al., 2004; Davidovich et al., 2008; Schuler et al., 2011). A study from Schneider's group showed that Skp2 mediates resistance of pancreatic cancer cells toward TRAIL (tumor necrosis factor-related apoptosis-inducing ligand)-induced apoptosis (Schuler et al., 2011). Ishii et al. (2004) reported that overexpression of Skp2 increased chemoresistance against camptothecin, cisplatin, and AG1478 in human lung cancer cells. In support of this notion, similar trend was also found in breast cancer. Davidovich et al. (2008) demonstrated that high preoperative expression of Skp2 was correlated with resistance to cyclophosphamide/doxorubicin/5-fluorouracil therapy in $94 \%$ of breast cancer patients. Interestingly, overexpression of Skp2 is not associated with resistance to docetaxel in breast cancer patients (Davidovich et al., 2008), suggesting that Skp2 expression may be a useful marker for predicting response to doxorubicin-based preoperative chemotherapy (Davidovich et al., 2008). However, Ravaioli et al. (2008) found that the relative effects of chemoendocrine versus endocrine therapy were similar regardless of the Skp2 expression status. Therefore, in order to better understand the precise role of Skp2 and its interrelationship with drug resistance, further in-depth investigations are required.

\section{Skp2 CROSS-TALKS WITH OTHER MAJOR SIGNALING PATHWAYS IN BREAST CANCER}

Although the molecular mechanism(s) by which Skp2 induces tumor growth has not been fully elucidated, multiple oncogenic pathways, such as mTOR, ERK, IGF-1, PPAR $\gamma$, PI3K/Akt, and FoxP3 have been reported to cross-talk with Skp2 (Lu et al., 2004; Lin and Yang, 2006; Shapira et al., 2006; Zaytseva et al., 2008; Gao et al., 2009a). Thus, cross-talks between Skp2 and other pathways could play critical roles in mammary tumorigenesis. Interestingly, recent findings suggest that the tumor suppressor function of BRCA1 depends on its BRCT (BRCA C terminus) phosphoprotein binding motif, but not its E3 ligase activity (Shakya et al., 2011). Recently it was reported that Skp2 could modulate the activity of p53 in an E3 ligase-independent manner (Kitagawa et al., 2008), indicating that Skp2 could exert its oncogenic function in both an E3 ligase-dependent and independent manner. In the following paragraphs, we will discuss some recent advances on the role of Skp2 in tumor progression, especially its cross-talk with other signaling pathways.

\section{THE ROLE OF mTOR PATHWAY IN THE REGULATION OF Skp2}

The cross-talk between mTOR pathway and Skp2 pathway has been reported recently (Pene et al., 2002; Shapira et al., 2006). It is known that mTOR plays an important role in the regulation of cellular homeostasis, cell growth, and survival pathways (Bjornsti and Houghton, 2004). In order to fulfill the regulatory function, mTOR kinase assembles into two distinct complexes (mTORC1 and mTORC2). The mTORC1 consists of mTOR, Raptor, proline-rich Akt substrate of $40 \mathrm{kDa}$ (PRAS40), and G-protein $\beta$-subunit-like protein $(\mathrm{G} \beta \mathrm{L})$. The well-characterized mTORC1 kinase substrates include S6K (p70 S6 ribosomal kinase) and phosphorylated 4E-binding protein 1 (4E-BP1; Bjornsti and Houghton, 
2004). The mTORC2 is composed of mTOR, Rictor, G $\beta L$, protein observed with Rictor-1 (PROTOR), and stress-activated protein kinase interacting protein 1 (Sin 1; Bjornsti and Houghton, 2004). Interestingly, the Raptor-containing complex is sensitive to rapamycin and regulates cell proliferation through phosphorylating $\mathrm{S} 6 \mathrm{~K}$ and $4 \mathrm{E}-\mathrm{BP} 1$, whereas the Rictor-containing complex is not sensitive to rapamycin (Hay and Sonenberg, 2004; Inoki et al., 2005).

Recently, the mTOR kinase has emerged as a critical player in the regulation of Skp2 (Shapira et al., 2006). Rapamycin, which inhibits the mTOR by directly binding the mTORC1, significantly decreased the expression of Skp2 both at mRNA and protein levels in a dose and time-dependent manner in breast cancer cell lines (Shapira et al., 2006). Moreover, it was found that negative effect of rapamycin on Skp2 expression has a critical role in rapamycin-mediated cell growth and G1 arrest (Shapira et al., 2006). Furthermore, rapamycin promoted the degradation of Skp2 and down-regulated the expression of the APC/C inhibitor Emi1 in breast cancer (Shapira et al., 2006), indicating that Skp2 could be a novel target for mediating the effects of rapamycin; however, the molecular mechanism by which mTOR regulates Skp2 remains to be elucidated.

\section{ERK AND ITS ROLE IN Skp2 SIGNALING}

Multiple studies have shown that ERK activities are up-regulated in many human cancers including breast cancer, and elevated ERK activity in human tumors has been correlated with poor prognosis, demonstrating that ERK may play a crucial role in human tumorigenesis (Kohno and Pouyssegur, 2006). It is known that ERK family includes ERK1 and ERK2, which belong to MAPK super family that regulates cell cycle progression, cell proliferation, differentiation, survival, and apoptosis (Kolch, 2005). Activation of MAPK requires dual phosphorylation by specific MAPK kinases (MKKs), and dephosphorylation by protein phosphatases, including MAPK phosphatases (MKPs; also known as dual-specificity phosphatase, DUSP; Seger and Krebs, 1995). It is well accepted that many different stimuli such as growth factors or carcinogens could activate the ERK pathway, leading to the activation of its targeted proteins such as downstream kinases and transcription factors that regulates the expression of specific genes and their activators/modulators (Roberts and Der, 2007; Shaul and Seger, 2007).

Recently, it has been reported that Skp2 is involved in ERK pathway (Lin and Yang, 2006). Lin and Yang (2006) verified that Skp2 participated in the ERK-directed ubiquitination and proteolysis of MKP-1, suggesting that ERK activity could be controlled via MKP-1 proteolysis in cooperation with Skp2. Consistent with this notion, suppression of Skp2 expression resulted in DUSP-1 up-regulation in human hepatocellular carcinoma (Calvisi et al., 2008). Furthermore, the cooperation between ERK and Skp2 has also been found in human breast cancer whereby synergistic activity of the two oncogenes has been shown to increase p27 degradation (Foster et al., 2003). Moreover, Foster et al. (2003) showed that estrogens elicit down-regulation of p27 through Skp2dependent and -independent mechanisms and could depend on p27 localization requiring the participation of other mediators of the Ras/Raf-1/ERK signaling pathway in breast cancer cells. However, it remains to be determined how Skp2 regulates the ERK activation in human breast cancers and the biological consequence of this regulation requires further investigation.

\section{PI 3K/Akt IN RELATION TO THE Skp2 PATHWAY}

The PI3K/Akt pathway has been reported to be involved in Skp2 pathway (Gao et al., 2009a). It has been well documented that Akt is a serine/threonine protein kinase, which is downstream of $\mathrm{PI} 3 \mathrm{~K}$ in response to growth factor stimulation (Hennessy et al., 2005). The Akt family of kinase includes three closely related isoforms designed as $\mathrm{Akt} 1 / \mathrm{PKB} \alpha$, Akt $2 / \mathrm{PKB} \beta$, and $\mathrm{Akt} 3 / \mathrm{PKB} \gamma$. Activated Akt promotes cell survival by suppressing apoptosis through promoting the phosphorylation and subsequent cytoplasmic localization of many downstream pro-apoptotic protein targets including Bad and Forkhead transcription factors such as FOXO1 and FOXO3a (Hennessy et al., 2005). In addition, Akt also modulates apoptosis indirectly by influencing the activities of several transcription factors such as NF- $\kappa$ B and CEBP (cyclic AMPresponsive element binding protein; Franke, 2008). Enhanced Akt signaling can also promote cell growth by inhibition of negative cell cycle regulators p21 and p27 (Franke, 2008). Thus, Akt has been believed to be a promising target for cancer prevention and treatment (Fresno Vara et al., 2004).

It has been reported that PI3K/Akt regulates p 27 expression via Skp2 in human cancer (Van Duijn and Trapman, 2006). Recently, we and other groups discovered that Akt controls Skp2 stability and the subcellular localization of Skp2 (Gao et al., 2009a; Lin et al., 2009). First of all, the positive correlation between Skp2 expression and Akt activity was found in a panel of breast cancer cell lines (Gao et al., 2009a). Moreover, inhibition of Aktl activity in breast cancer cells caused down-regulation of Skp2 expression, indicating that elevated Akt activity could be one major cause of the observed up-regulation of Skp2 in breast cancer (Gao et al., 2009a). Further research toward exploration of the molecular mechanisms by which Akt promotes cytoplasmic localization of Skp2 still requires further attention.

\section{IGF AND ITS CROSS-TALK WITH THE Skp2 PATHWAY}

It is widely accepted that IGF-1 could bind to its receptors including IGF-1 receptor (IGF1R) and insulin receptor. In addition, IGF-1 has been shown to bind and interact with the IGFBPs (IGF1 binding proteins), of which there are six identified members (IGFBP1-6) so far (Grimberg, 2003). After binding to the IGF1R, IGF-1 initiates cellular signaling such as activation of PI3K/Akt signaling pathway and/or Ras/Raf/MAPK pathway, which influences cell proliferation, survival, and apoptosis (Clayton et al., 2011). Therefore, the increased level of IGF-1 promotes cell proliferation, inhibits apoptosis, and enhances angiogenesis, all are involved in the development and progression of human cancers (Heidegger et al., 2011). A recent study has shown that IGF1 regulates the expression of Skp2 in breast cancer (Lu et al., 2004). Lu and colleagues found that IGF-1 decreased p27 expression via up-regulation of Skp2. Moreover, chemotherapeutic drug trastuzumab (also known as Herceptin) decreased the expression level of Skp2, whereas this decrease was attenuated by IGF-1 in various breast cancer cells (Lu et al., 2004). Furthermore, IGF1-mediated reduction in $\mathrm{p} 27$ protein mediated via increased Skp2 expression involves the activation of PI3K pathway, suggesting that 
Skp2 could be an attractive target for the treatment of human cancer by attenuating multiple other growth signaling pathways.

\section{PPAR $\gamma$ AND ITS RELATIONSHIP WITH Skp2}

Peroxisome proliferator-activated receptor- $\gamma$, a ligand-activated transcription factor, has been demonstrated to provoke and mediate anti-inflammatory signaling (Schmidt et al., 2010). PPAR $\gamma$ binds to PPREs (peroxisome proliferators response elements) as a heterodimer with members of the RXR (retinoid X receptor) subfamily, leading to the regulation of target gene expression (Schmidt et al., 2010). It is widely accepted that PPAR $\gamma$ is a tumor suppressor protein because it initiates a number of anti-neoplastic processes such as arresting the cell cycle, causing cell differentiation, inhibition of angiogenesis as well as induction of apoptosis (Schmidt et al., 2010). It has been observed that PPAR $\gamma$ induced the expression of PTEN in breast cancer cell lines, possibly by binding to putative PPREs, resulting in Akt phosphorylation and reduced cell proliferation (Patel et al., 2001; Bonofiglio et al., 2005). However, one study demonstrated that down-regulation of PPAR $\gamma$ suppressed cell growth and induced apoptosis in MCF-7 breast cancer cells (Zaytseva et al., 2008), arguing that the tumor suppressive role of PPAR $\gamma$ could be cell-type or cellular context dependent.

Recently, PPAR $\gamma$ has been found to regulate Skp2 expression in human cancers including breast cancer (Wei et al., 2007; Meng et al., 2010). For example, thiazolidinediones (TZD), an agonist of PPAR $\gamma$, have been found to down-regulate Skp2 with accumulation of its substrate p27 in prostate cancer (Wei et al., 2007). More recently, PPAR $\gamma$ overexpression was reported to suppress Skp2 levels. More importantly, the inverse correlation between the expression of PPAR $\gamma$ and Skp2 was identified in both breast cancer cell lines and human breast cancer specimens (Meng et al., 2010). Consistent with this, PPAR $\gamma$ overexpression inhibited the expression of Skp2 at both mRNA and protein levels, leading to reduced cell proliferation and induction of apoptosis (Meng et al., 2010). Furthermore, overexpression of Skp2 partially abrogates PPAR $\gamma$ 's pro-apoptotic and anti-proliferative abilities (Meng et al., 2010), suggesting that anti-neoplastic role of PPAR $\gamma$ could in part be mediated through deregulation of Skp2 expression in breast cancer. However, more studies are required to fully understand how $\operatorname{PPAR} \gamma$ regulates Skp2 signaling pathway in human breast cancer.

\section{THE ROLE OF FoxP3 WITH RESPECT TO SkP2 SIGNALING}

The FoxP3 (Forkhead box P3), an X-linked tumor suppressor gene, is a member of the forkhead box/winged-helix transcription factor family (Wang et al., 2010a). FoxP3 is highly expressed in regulatory $\mathrm{T}$ cells, behaving as a master regulator in the development and differentiation of regulatory $\mathrm{T}$ cells. In recent years, it has been shown that FoxP3 is frequently inactivated in many human cancers such as prostate cancer, ovarian cancer, and breast cancer (Zuo et al., 2007b; Wang et al., 2009; Zhang and Sun, 2010). For example, overexpression of FoxP3 inhibited cell proliferation, decreased cell migration, and reduced cell invasion in ovarian cancer (Zhang and Sun, 2010). In addition, FoxP3 retarded prostate tumorigenesis through inhibition of oncogene Myc (Wang et al., 2009). Moreover, FoxP3 has been identified as a transcriptional repressor of the HER2 oncogene (Zuo et al., 2007b). Interestingly, FoxP3 also suppressed growth and induced cell death in breast cancer cell line without HER2 overexpression (Zuo et al., 2007b), indicating that FoxP3 may affect other pathways that are involved in breast cancer. Indeed, Zhang et al. found that FoxP3 is a novel transcriptional repressor of Skp2 in human breast cancer (Zuo et al., 2007a). Overexpression of FoxP3 inhibited Skp2 expression with increased p 27 in breast cancer cells, while down-regulation of FoxP3 in human mammary epithelial cells increased Skp2 levels (Zuo et al., 2007a). This finding has been further supported by the observed inverse correlation between FoxP3 and Skp2 levels in primary breast cancer samples (Zuo et al., 2007a). Moreover, down-regulation of Skp2 was critical for FoxP3-mediated growth inhibition in breast cancer cells (Zuo et al., 2007a), demonstrating that FoxP3 is a Skp2 repressor in breast cancer. However, how FoxP3 regulates Skp2 expression and whether this regulation could be exploited for fighting the battle against breast cancer will require further in-depth investigations.

\section{Skp2 INHIBITION IS A NOVEL STRATEGY FOR BREAST CANCER TREATMENT}

Since Skp2 is frequently amplified and overexpressed in human breast cancer, Skp2 could be a potential molecular target for breast cancer therapy (Ohta and Fukuda, 2004). Therefore, inhibition of Skp2 may be a novel strategy for the prevention and/or treatment of breast cancer. To this end, several small molecule inhibitors to block Skp2 expression have been developed by a highthroughput screening (Chen et al., 2008; Rico-Bautista et al., 2010). For example, a small molecular inhibitor CpdA (Compound A), which blocks the recruitment of Skp2 to the SCF ligase, caused cell cycle arrest, cell growth inhibition, and apoptosis in multiple myeloma cells (Chen et al., 2008). Moreover, one chemical compound known as SMIP0004 was found to down-regulate Skp2 and subsequently caused p27 stabilization in prostate cancer cells (Rico-Bautista et al., 2010). Unfortunately, specific drugs that inactivate Skp2 in breast cancer are unavailable so far, although we believe that there is renewed interest in developing Skp2 inhibitors for breast cancer treatment.

It is noteworthy that several natural compounds has been found to down-regulate Skp2 expression in human cancers including breast cancer (Huang et al., 2011). For example, curcumin, lycopene, pentagalloylglucose, and quercetin inhibited Skp2 expression, leading to cell growth inhibition and cell cycle arrest through increased FOXO1 in breast cancer cells (Huang et al., 2011). ATRA (all-trans retinoic acid) promoted the ubiquitination of Skp2 in breast cancer cell lines, leading to cell cycle arrest (Dow et al., 2001). Consistent with this notion, overexpression of Skp2 promotes resistance to ATRA and prevents p27 accumulation in breast cancer cells (Dow et al., 2001). Hsu et al. (2011) found that gallic acid markedly reduced cell growth of human breast cancer cells and induced cell cycle arrest by inhibition of Skp2 and attenuation of Skp2-p27 association as well as reduction of p27 ubiquitination. Huang et al. (2008) reported that EGCG (Epigallocatechin-3-gallate), the main constituent of green tea, inhibited human breast cancer cell growth in part through down-regulation of Skp2 expression and accumulation of p27. Furthermore, both tamoxifen and paclitaxel significantly and synergistically enhanced cell growth inhibition by EGCG mediated through the down-regulation of Skp2 expression in breast cancer 
cells (Huang et al., 2008). Interestingly, the inhibition of Skp2 was not always correlated with increased p27 expression, indicating that EGCG-dependent Skp2 down-regulation could reduce cell growth via other downstream pathway(s) (Huang et al., 2008). These published data provides strong evidence in support of the idea that inhibition of Skp2 may be a promising therapeutic strategy for the treatment of human breast cancer.

\section{CONCLUSION AND OVERALL PERSPECTIVES}

In this short review article, we have provided succinct information as to the state of our knowledge on the role of Skp2 in human breast cancer. However, it is worth mentioning that the critical roles for Skp2 as an oncoprotein in human cancer progression are largely uncertain, although studies establishing the relationship between Skp2 and cancer have been burst onto the scene in recent years, and several groups have found that multiple genes including PI3K/Akt, ERK, mTOR, and FoxP3, could regulate the Skp2 expression (Figure 2). Skp2 has been revealed as a novel target for the prevention and/or treatment of human cancers including but not limited to breast cancer. Therefore, the development of agents for specifically targeting Skp2 is likely to have a significant therapeutic impact on the treatment of human cancers. Furthermore, here we summarized that several compounds, especially natural compounds that could specifically target Skp2 may act as anticancer drugs. Due to non-toxic features, inhibition of Skp2 by natural agents could be a novel and safer approach for empowering anticancer therapy. However, further pre-clinical studies are needed to find the right combinations with chemotherapeutic drugs toward better treatment of human mammary malignancies. We hope that this article could promote further study for the development of specific inhibitors/antagonists for targeted inactivation of Skp2 for cancer therapy by either single agent or by using a combinational approach. In conclusion, targeted inactivation of Skp2 would likely become a novel newer strategy for the prevention of tumor progression and/or successful treatment of human malignancies including breast cancer in the future.

\section{REFERENCES}

Amati, B., and Vlach, J. (1999). Kipl meets SKP2: new links in cellcycle control. Nat. Cell Biol. 1, E91-E93.

Bashir, T., and Pagano, M. (2004). Don't skip the G1 phase: how APC/CCdh1 keeps SCFSKP2 in check. Cell Cycle 3, 850-852.

Bhattacharya, S., Garriga, J., Calbo, J., Yong, T., Haines, D. S., and Grana, X. (2003). SKP2 associates with p130 and accelerates p130 ubiquitylation and degradation in human cells. Oncogene 22, 2443-2451.

Bjornsti, M. A., and Houghton, P. J. (2004). The TOR pathway: a target for cancer therapy. Nat. Rev. Cancer 4, 335-348.

Bonofiglio, D., Gabriele, S., Aquila, S., Catalano, S., Gentile, M., Middea, E., Giordano, F., and Ando, S. (2005). Estrogen receptor alpha binds to peroxisome proliferatoractivated receptor response element and negatively interferes with peroxisome proliferator-activated receptor gamma signaling in breast cancer cells. Clin. Cancer Res. 11, 6139-6147.

Calvisi, D. F., Pinna, F., Meloni, F., Ladu, L., Simile, M. M., De Miglio, M. R., Virdis, P., Frau, M., Tomasi, M. L., Seddaiu, M. A., Muroni, M. R., Feo, F., and Pascale, R. M. (2008). Dual-specificity phosphatase 1 ubiquitination in extracellular signalregulated kinase-mediated control of growth in human hepatocellular carcinoma. Cancer Res. 68, 4192-4200.

Carrano, A. C., Eytan, E., Hershko, A., and Pagano, M. (1999). SKP2 is required for ubiquitinmediated degradation of the CDK S., Pellegrino, R., Sini, M., Daino,

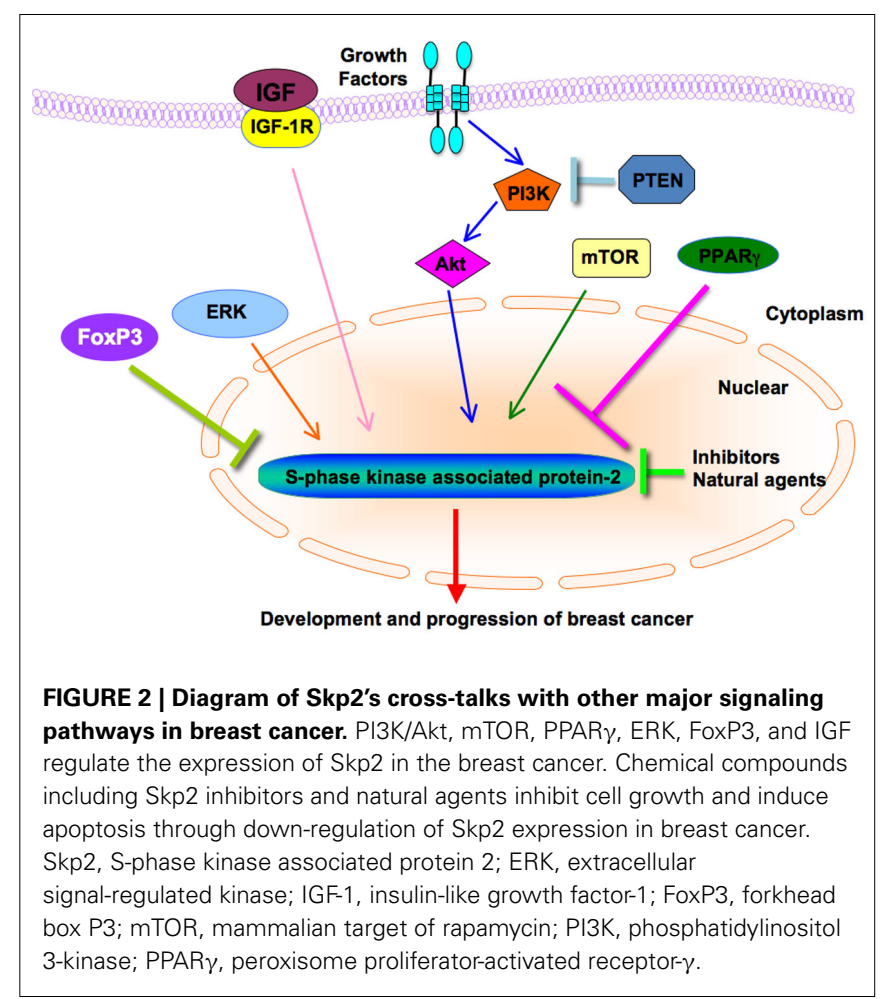

\section{ACKNOWLEDGMENTS}

We sincerely apologize to those authors whose work could not be cited in this succinct review article because of the space limitation. The authors' work cited in this review was funded by grants from the National Institute of General Medicines, NIH (GM089763) to Wenyi Wei, and Massachusetts Life Science Center New Investigator award (Wenyi Wei), and Department of Defense Prostate New Investigator award to Wenyi Wei. Zhiwei Wang is supported by NRSA fellowship. Wenyi Wei is an ACS scholar.

inhibitor p27. Nat. Cell Biol. 1, 193-199.

Chan, C. H., Lee, S. W., Li, C. F., Wang, J., Yang, W. L., Wu, C. Y., Wu, J., Nakayama, K. I., Kang, H. Y., Huang, H. Y., Hung, M. C., Pandolfi, P. P., and Lin, H. K. (2010a). Deciphering the transcriptional complex critical for RhoA gene expression and cancer metastasis. Nat. Cell Biol. 12, 457-467.

Chan, C. H., Lee, S. W., Wang, J., and Lin, H. K. (2010b). Regulation of Skp2 expression and activity and its role in cancer progression. Sci. World J. 10, 1001-1015.

Chander, H., Halpern, M., ResnickSilverman, L., Manfredi, J. J., and Germain, D. (2010). Skp2B attenuates 553 function by inhibiting prohibitin. EMBO Rep. 11, 220-225.

Chen, Q., Xie, W., Kuhn, D. J., Voorhees, P. M., Lopez-Girona, A., Mendy, D.,
Corral, L. G., Krenitsky, V. P., Xu, W., Moutouh-De Parseval, L., Webb, D. R., Mercurio, F., Nakayama, K. I., Nakayama, K., and Orlowski, R. Z. (2008). Targeting the p27 E3 ligase $\mathrm{SCF}(\mathrm{Skp} 2)$ results in $\mathrm{p} 27$ - and Skp2-mediated cell-cycle arrest and activation of autophagy. Blood 111, 4690-4699.

Clayton, P. E., Banerjee, I., Murray, P. G., and Renehan, A. G. (2011). Growth hormone, the insulin-like growth factor axis, insulin and cancer risk. Nat. Rev. Endocrinol. 7, 11-24.

Davidovich, S., Ben-Izhak, O., Shapira, M., Futerman, B., and Hershko, D. D. (2008). Over-expression of Skp2 is associated with resistance to preoperative doxorubicin-based chemotherapy in primary breast cancer. Breast Cancer Res. 10, R63. 
Dow, R., Hendley, J., Pirkmaier, A., Musgrove, E. A., and Germain, D. (2001). Retinoic acid-mediated growth arrest requires ubiquitylation and degradation of the F-box protein Skp2. J. Biol. Chem. 276, 45945-45951.

Einama, T., Kagata, Y., Tsuda, H., Morita, D., Ogata, S., Ueda, S., Takigawa, T., Kawarabayashi, N., Fukatsu, K., Sugiura, Y., Matsubara, O., and Hatsuse, K. (2006). High-level Skp2 expression in pancreatic ductal adenocarcinoma: correlation with the extent of lymph node metastasis, higher histological grade, and poorer patient outcome. Pancreas 32, 376-381.

Fang, F. M., Chien, C. Y., Li, C. F., Shiu, W. Y., Chen, C. H., and Huang, H. Y. (2009). Effect of S-phase kinaseassociated protein 2 expression on distant metastasis and survival in nasopharyngeal carcinoma patients. Int. J. Radiat. Oncol. Biol. Phys. 73, 202-207.

Foley, J., Nickerson, N. K., Nam, S., Allen, K. T., Gilmore, J. L., Nephew, K. P., and Riese, D. J. II. (2010). EGFR signaling in breast cancer: bad to the bone. Semin. Cell Dev. Biol. 21, 951-960.

Foster, J. S., Fernando, R. I., Ishida, N., Nakayama, K. I., and Wimalasena, J. (2003). Estrogens down-regulate p27Kip1 in breast cancer cells through Skp2 and through nuclear export mediated by the ERK pathway. J. Biol. Chem. 278, 41355-41366.

Franke, T. F. (2008). PI3K/Akt: getting it right matters. Oncogene 27, 6473-6488.

Frescas, D., and Pagano, M. (2008). Deregulated proteolysis by the F-box proteins SKP2 and beta-TrCP: tipping the scales of cancer. Nat. Rev. Cancer 8, 438-449.

Fresno Vara, J. A., Casado, E., De Castro, J., Cejas, P., Belda-Iniesta, C., and Gonzalez-Baron, M. (2004). PI3K/Akt signalling pathway and cancer. Cancer Treat. Rev. 30, 193-204.

Fujita, T., Liu, W., Doihara, H., Date, H., and Wan, Y. (2008). Dissection of the APCCdh1-Skp2 cascade in breast cancer. Clin. Cancer Res. 14, 1966-1975.

Gao, D., Inuzuka, H., Tseng, A., Chin, R. Y., Toker, A., and Wei, W. (2009a). Phosphorylation by Akt1 promotes cytoplasmic localization of Skp2 and impairs APCCdh1mediated Skp2 destruction. Nat. Cell Biol. 11, 397-408.

Gao, D., Inuzuka, H., Tseng, A., and Wei, W. (2009b). Akt finds its new path to regulate cell cycle through modulating Skp2 activity and its destruction by APC/Cdh1. Cell Div. 4, 11.

Grimberg, A. (2003). Mechanisms by which IGF-I may promote cancer. Cancer Biol. Ther. 2, 630-635.

Gstaiger, M., Jordan, R., Lim, M., Catzavelos, C., Mestan, J., Slingerland, J., and Krek, W. (2001). Skp2 is oncogenic and overexpressed in human cancers. Proc. Natl. Acad. Sci. U.S.A. 98, 5043-5048.

Guo, S., Liu, M., and Gonzalez-Perez, R. R. (2011). Role of Notch and its oncogenic signaling crosstalk in breast cancer. Biochim. Biophys. Acta $1815,197-213$.

Haagenson, K. K., and Wu, G. S. (2010). The role of MAP kinases and MAP kinase phosphatase-1 in resistance to breast cancer treatment. Cancer Metastasis Rev. 29, 143-149.

Hay, N., and Sonenberg, N. (2004). Upstream and downstream of mTOR. Genes Dev. 18, 1926-1945.

Heidegger, I., Pircher, A., Klocker, H., and Massoner, P. (2011). Targeting the insulin-like growth factor network in cancer therapy. Cancer Biol. Ther. 11, 701-707.

Hennessy, B. T., Smith, D. L., Ram, P. T., Lu, Y., and Mills, G. B. (2005). Exploiting the PI3K/AKT pathway for cancer drug discovery. Nat. Rev. Drug Discov. 4, 988-1004.

Hernandez-Aya, L. F., and GonzalezAngulo, A. M. (2011). Targeting the phosphatidylinositol 3-kinase signaling pathway in breast cancer. Oncologist 16, 404-414.

Hershko, D. D., and Shapira, M. (2006). Prognostic role of p27Kip1 deregulation in colorectal cancer. Cancer 107, 668-675.

Hiramatsu, Y., Kitagawa, K., Suzuki, T., Uchida, C., Hattori, T., Kikuchi, H., Oda, T., Hatakeyama, S., Nakayama, K. I., Yamamoto, T., Konno, H., and Kitagawa, M. (2006). Degradation of Tob1 mediated by SCFSkp2dependent ubiquitination. Cancer Res. 66, 8477-8483.

Hsu, J. D., Kao, S. H., Ou, T. T., Chen, Y. J., Li, Y. J., and Wang, C. J. (2011). Gallic acid induces G2/M phase arrest of breast cancer cell MCF-7 through stabilization of p27(Kip1) attributed to disruption of p27(Kip1)/Skp2 complex. J. Agric. Food Chem. 59, 1996-2003.

Huang, H., Regan, K. M., Wang, F., Wang, D., Smith, D. I., Van Deursen, J. M., and Tindall, D. J. (2005). Skp2 inhibits FOXO1 in tumor suppression through ubiquitin-mediated degradation. Proc. Natl. Acad. Sci. U.S.A. 102, 1649-1654.
Huang, H. C., Lin, C. L., and Lin J. K. (2011). 1,2,3,4,6-penta-Ogalloyl-beta-D-glucose, quercetin, curcumin and lycopene induce cell-cycle arrest in MDA-MB-231 and BT474 cells through downregulation of Skp2 protein. J. Agric. Food Chem. 59, 6765-6775.

Huang, H. C., Way, T. D., Lin, C. L., and Lin, J. K. (2008). EGCG stabilizes p27kip1 in E2-stimulated MCF7 cells through down-regulation of the Skp2 protein. Endocrinology 149, 5972-5983.

Hulit, J., Lee, R. J., Li, Z., Wang, C., Katiyar, S., Yang, J., Quong, A. A., Wu, K., Albanese, C., Russell, R., Di Vizio, D., Koff, A., Thummala, S., Zhang, H., Harrell, J., Sun, H., Muller, W. J., Inghirami, G., Lisanti, M. P., and Pestell, R. G. (2006). p27Kip1 repression of ErbB2-induced mammary tumor growth in transgenic mice involves Skp2 and Wnt/betacatenin signaling. Cancer Res. 66 , 8529-8541.

Hung, W. C., Tseng, W. L., Shiea, J., and Chang, H. C. (2010). Skp2 overexpression increases the expression of MMP-2 and MMP-9 and invasion of lung cancer cells. Cancer Lett. 288, 156-161.

Inoki, K., Corradetti, M. N., and Guan, K. L. (2005). Dysregulation of the TSC-mTOR pathway in human disease. Nat. Genet. 37, 19-24.

Ishii, T., Matsuse, T., Masuda, M. and Teramoto, S. (2004). The effects of S-phase kinase-associated protein 2 (SKP2) on cell cycle status, viability, and chemoresistance in A549 lung adenocarcinoma cells. Exp. Lung Res. 30 687-703.

Kamura, T., Hara, T., Kotoshiba, S., Yada, M., Ishida, N., Imaki, H., Hatakeyama, S., Nakayama, K., and Nakayama, K. I. (2003). Degradation of p57Kip2 mediated by SCFSkp2-dependent ubiquitylation. Proc. Natl. Acad. Sci. U.S.A. 100 10231-10236

Kitagawa, M., Lee, S. H., and Mccormick, F. (2008). Skp2 suppresses p53-dependent apoptosis by inhibiting p300. Mol. Cell 29, 217-231.

Kohno, M., and Pouyssegur, J. (2006). Targeting the ERK signaling pathway in cancer therapy. Ann. Med. 38, 200-211.

Kolch, W. (2005). Coordinating ERK/MAPK signalling through scaffolds and inhibitors. Nat. Rev. Mol. Cell Biol. 6, 827-837.

Lee, S. H., and McCormick, F. (2005). Downregulation of Skp2 and p27/Kip1 synergistically induces apoptosis in T98G glioblastoma cells. J. Mol. Med. 83, 296-307.

Li, J. Q., Wu, F., Masaki, T., Kubo, A., Fujita, J., Dixon, D. A., Beauchamp, R. D., Ishida, T., Kuriyama, S., and Imaida, K. (2004). Correlation of Skp2 with carcinogenesis, invasion, metastasis, and prognosis in colorectal tumors. Int. J. Oncol. 25, 87-95.

Lin, H. K., Wang, G., Chen, Z., TeruyaFeldstein, J., Liu, Y., Chan, C. H., Yang, W. L., Erdjument-Bromage, H., Nakayama, K. I., Nimer, S. Tempst, P., and Pandolfi, P. P. (2009). Phosphorylation-dependent regulation of cytosolic localization and oncogenic function of $\mathrm{Skp} 2$ by Akt/PKB. Nat. Cell Biol. 11, 420-432.

Lin, S. X., Chen, J., Mazumdar, M., Poirier, D., Wang, C., Azzi, A., and Zhou, M. (2010). Molecular therapy of breast cancer: progress and future directions. Nat. Rev. Endocrinol. 6, 485-493.

Lin, Y. W., and Yang, J. L. (2006). Cooperation of ERK and SCFSkp2 for MKP-1 destruction provides a positive feedback regulation of proliferating signaling. J. Biol. Chem. 281, 915-926.

Lu, M., Ma, J., Xue, W., Cheng, C., Wang, Y., Zhao, Y., Ke, Q., Liu, H., Liu, Y., Li, P., Cui, X., He, S., and Shen, A. (2009). The expression and prognosis of FOXO3a and Skp2 in human hepatocellular carcinoma. Pathol. Oncol. Res. 15, 679-687.

Lu, Y., Zi, X., and Pollak, M. (2004). Molecular mechanisms underlying IGF-I-induced attenuation of the growth-inhibitory activity of trastuzumab (Herceptin) on SKBR3 breast cancer cells. Int. J. Cancer 108, 334-341.

Ma, X. M., Liu, Y., Guo, J. W., Liu, J. H., and Zuo, L. F. (2005). Relation of overexpression of S phase kinaseassociated protein 2 with reduced expression of p27 and PTEN in human gastric carcinoma. World J. Gastroenterol. 11, 6716-6721.

Meng, J., Ding, Y., Shen, A., Yan, M., He, F., Ji, H., Zou, L., Liu, Y., Wang, Y., Lu, X., and Wang, H. (2010). Overexpression of PPARgamma can down-regulate $\mathrm{Skp} 2$ expression in MDA-MB-231 breast tumor cells. Mol. Cell. Biochem. 345, 171-180.

Michel, J. J., and Xiong, Y. (1998). Human CUL-1, but not other cullin family members, selectively interacts with SKP1 to form a complex with SKP2 and cyclin A. Cell Growth Differ. 9, 435-449.

Nahta, R., and O'Regan, R. M. (2010). Evolving strategies for overcoming resistance to HER2-directed therapy: targeting the PI3K/Akt/mTOR 
pathway. Clin. Breast Cancer 10(Suppl. 3), S72-S78.

Nakayama, K., Nagahama, H., Minamishima, Y. A., Matsumoto, M., Nakamichi, I., Kitagawa, K., Shirane, M., Tsunematsu, R., Tsukiyama, T., Ishida, N., Kitagawa, M., and Hatakeyama, S. (2000). Targeted disruption of Skp2 results in accumulation of cyclin E and p27 (Kip1), polyploidy and centrosome overduplication. EMBO J. 19, 2069-2081.

Nakayama, K. I., and Nakayama, K. (2006). Ubiquitin ligases: cell-cycle control and cancer. Nat. Rev. Cancer 6, 369-381.

Nalepa, G., Rolfe, M., and Harper, J. W. (2006). Drug discovery in the ubiquitin-proteasome system. Nat. Rev. Drug Discov. 5, 596-613.

Narod, S. A. (2010). BRCA mutations in the management of breast cancer: the state of the art. Nat. Rev. Clin. Oncol. 7, 702-707.

Ohta, T., and Fukuda, M. (2004). Ubiquitin and breast cancer. Oncogene 23, 2079-2088.

Okamoto, Y., Ozaki, T., Miyazaki, K., Aoyama, M., Miyazaki, M., and Nakagawara, A. (2003). UbcH10 is the cancer-related E2 ubiquitinconjugating enzyme. Cancer Res. 63, 4167-4173.

O'Regan, R., and Hawk, N. N. (2011). mTOR inhibition in breast cancer: unraveling the complex mechanisms of mTOR signal transduction and its clinical implications in therapy. Expert Opin. Ther. Targets 15, 859-872.

Patel, L., Pass, I., Coxon, P., Downes, C. P., Smith, S. A., and Macphee, C. H. (2001). Tumor suppressor and anti-inflammatory actions of PPARgamma agonists are mediated via upregulation of PTEN. Curr. Biol. 11, 764-768.

Pene, F., Claessens, Y. E., Muller, O., Viguie, F., Mayeux, P., Dreyfus, F., Lacombe, C., and Bouscary, D. (2002). Role of the phosphatidylinositol 3-kinase/Akt and mTOR/P70S6-kinase pathways in the proliferation and apoptosis in multiple myeloma. Oncogene 21, 6587-6597.

Prosperi, J. R., and Goss, K. H. (2010). A Wnt-ow of opportunity: targeting the Wnt/beta-catenin pathway in breast cancer. Curr. Drug Targets 11, 1074-1088.

Radke, S., Pirkmaier, A., and Germain, D. (2005). Differential expression of the F-box proteins Skp2 and Skp2B in breast cancer. Oncogene 24, 3448-3458.
Ravaioli, A., Monti, F., Regan, M. M., Maffini, F., Mastropasqua, M. G., Spataro, V., Castiglione-Gertsch, M., Panzini, I., Gianni, L., Goldhirsch, A., Coates, A., Price, K. N., Gusterson, B. A., and Viale, G. (2008). p27 and Skp2 immunoreactivity and its clinical significance with endocrine and chemo-endocrine treatments in node-negative early breast cancer. Ann. Oncol. 19, 660-668.

Rico-Bautista, E., Yang, C. C., Lu, L., Roth, G. P., and Wolf, D. A. (2010). Chemical genetics approach to restoring p27Kip1 reveals novel compounds with antiproliferative activity in prostate cancer cells. BMC Biol. 8, 153. doi:10.1186/1741-7007-8-153

Roberts, P. J., and Der, C. J. (2007). Targeting the Raf-MEK-ERK mitogenactivated protein kinase cascade for the treatment of cancer. Oncogene 26, 3291-3310.

Rose, A. E., Wang, G., Hanniford, D., Monni, S., Tu, T., Shapiro, R. L., Berman, R. S., Pavlick, A. C., Pagano, M., Darvishian, F., Mazumdar, M., Hernando, E., and Osman, I. (2011). Clinical relevance of SKP2 alterations in metastatic melanoma. Pigment Cell Melanoma Res. 24, 197-206.

Rosen, L. S., Ashurst, H. L., and Chap, L. (2010). Targeting signal transduction pathways in metastatic breast cancer: a comprehensive review. Oncologist 15, 216-235.

Schmidt, M. V., Brune, B., and Von Knethen,A. (2010). The nuclear hormone receptor PPARgamma as a therapeutic target in major diseases. Sci. World J. 10, 2181-2197.

Schuler, S., Diersch, S., Hamacher, R., Schmid, R. M., Saur, D., and Schneider, G. (2011). SKP2 confers resistance of pancreatic cancer cells towards TRAIL-induced apoptosis. Int. J. Oncol. 38, 219-225.

Seger, R., and Krebs, E. G. (1995). The MAPK signaling cascade. FASEB J. 9, 726-735.

Seki, R., Okamura, T., Koga, H., Yakushiji, K., Hashiguchi, M., Yoshimoto, K., Ogata, H., Imamura, R., Nakashima, Y., Kage, M., Ueno, T., and Sata, M. (2003). Prognostic significance of the F-box protein Skp2 expression in diffuse large Bcell lymphoma. Am. J. Hematol. 73, 230-235.

Shakya, R., Reid, L. J., Reczek, C. R., Cole, F., Egli, D., Lin, C. S., Derooij, D. G., Hirsch, S., Ravi, K., Hicks, J. B., Szabolcs, M., Jasin, M., Baer, R., and Ludwig, T. (2011). BRCA1 tumor suppression depends on BRCT phosphoprotein binding, but not its E3 ligase activity. Science 334, 525-528.

Shapira, M., Kakiashvili, E., Rosenberg, T., and Hershko, D. D. (2006). The mTOR inhibitor rapamycin downregulates the expression of the ubiquitin ligase subunit Skp2 in breast cancer cells. Breast Cancer Res. 8 , R46.

Shaul, Y. D., and Seger, R. (2007) The MEK/ERK cascade: from signaling specificity to diverse functions. Biochim. Biophys. Acta 1773, 1213-1226.

Siegel, R., Ward, E., Brawley, O., and Jemal, A. (2011). Cancer statistics, 2011: the impact of eliminating socioeconomic and racial disparities on premature cancer deaths. $C A$ Cancer J. Clin. 61, 212-236.

Signoretti, S., Di Marcotullio, L., Richardson, A., Ramaswamy, S., Isaac, B., Rue, M., Monti, F., Loda, M., and Pagano, M. (2002). Oncogenic role of the ubiquitin ligase subunit Skp2 in human breast cancer. $J$. Clin. Invest. 110, 633-641.

Skaar, J. R., and Pagano, M. (2009). Control of cell growth by the SCF and APC/C ubiquitin ligases. Curr. Opin. Cell Biol. 21, 816-824

Sonoda, H., Inoue, H., Ogawa, K., Utsunomiya, T., Masuda, T. A., and Mori, M. (2006). Significance of $\mathrm{skp} 2$ expression in primary breast cancer. Clin. Cancer Res. 12, 1215-1220.

Sun, L., Cai, L., Yu, Y., Meng, Q., Cheng, X., Zhao, Y., Sui, G., and Zhang, F. (2007). Knockdown of S-phase kinase-associated protein-2 expression in MCF-7 inhibits cell growth and enhances the cytotoxic effects of epirubicin. Acta Biochim. Biophys. Sin. (Shanghai) 39, 999-1007.

Tedesco, D., Lukas, J., and Reed, S. I. (2002). The pRb-related protein p130 is regulated by phosphorylation-dependent proteolysis via the protein-ubiquitin ligase SCF (Skp2). Genes Dev. 16, 2946-2957.

Tosco, P., La Terra Maggiore, G. M., Forni, P., Berrone, S., Chiusa, L., and Garzino-Demo, P. (2011). Correlation between Skp2 expression and nodal metastasis in stage I and II oral squamous cell carcinomas. Oral. Dis. 17, 102-108.

Tsvetkov, L. M., Yeh, K. H., Lee, S. J., Sun, H., and Zhang, H. (1999). p27(Kip1) ubiquitination and degradation is regulated by the $\mathrm{SCF}$ (Skp2) complex through phosphorylated Thr187 in p27. Curr. Biol. 9, 661-664.

Umanskaya, K., Radke, S., Chander, H., Monardo, R., Xu, X., Pan, Z. Q., O'Connell, M. J., and Germain,
D. (2007). Skp2B stimulates mammary gland development by inhibiting REA, the repressor of the estrogen receptor. Mol. Cell. Biol. 27, 7615-7622.

Van Duijn, P. W., and Trapman, J. (2006). PI3K/Akt signaling regulates p27 (kip1) expression via Skp2 in PC3 and DU145 prostate cancer cells, but is not a major factor in p27 (kipl) regulation in $\mathrm{LNCaP}$ and PC346 cells. Prostate 66, 749-760.

Visbal, A. P., and Lewis, M. T. (2010). Hedgehog signaling in the normal and neoplastic mammary gland. Curr. Drug Targets 11, 1103-1111.

Voduc, D., Nielsen, T. O., Cheang, M. C., and Foulkes, W. D. (2008). The combination of high cyclin E and Skp2 expression in breast cancer is associated with a poor prognosis and the basal phenotype. Hum. Pathol. 39, 1431-1437.

Wang, L., Liu, R., Li, W., Chen, C., Katoh, H., Chen, G. Y., Mcnally, B., Lin, L., Zhou, P., Zuo, T., Cooney, K. A., Liu, Y., and Zheng, P. (2009). Somatic single hits inactivate the $\mathrm{X}$-linked tumor suppressor FOXP3 in the prostate. Cancer Cell 16, 336-346.

Wang, L., Liu, R., Ribick, M., Zheng, P., and Liu, Y. (2010a). FOXP3 as an X-linked tumor suppressor. Discov. Med. 10, 322-328.

Wang, Z., Li, Y., Ahmad, A., Azmi, A. S., Kong, D., Banerjee, S., and Sarkar, F. H. (2010b). Targeting miRNAs involved in cancer stem cell and EMT regulation: an emerging concept in overcoming drug resistance. Drug Resist. Updat. 13, 109-118.

Wang, Z., Gao, D., Fukushima, H., Inuzuka, H., Liu, P., Wan, L., Sarkar, F. H., and Wei, W. (2011a). Skp2: a novel potential therapeutic target for prostate cancer. Biochim. Biophys. Acta 1825, 11-17.

Wang, Z., Li, Y., Ahmad, A., Banerjee, S., Azmi, A. S., Kong, D., and Sarkar, F. H. (2011b). Pancreatic cancer: understanding and overcoming chemoresistance. Nat. Rev. Gastroenterol. Hepatol. 8, 27-33.

Wei, S., Lin, L. F., Yang, C. C., Wang, Y. C., Chang, G. D., Chen, H., and Chen, C. S. (2007). Thiazolidinediones modulate the expression of beta-catenin and other cell-cycle regulatory proteins by targeting the F-box proteins of Skp1-Cull-F-box protein E3 ubiquitin ligase independently of peroxisome proliferator-activated receptor gamma. Mol. Pharmacol. 72, 725-733.

Weissman, A. M., Shabek, N., and Ciechanover, A. (2011). The predator becomes the prey: regulating the ubiquitin system by ubiquitylation 
and degradation. Nat. Rev. Mol. Cell Biol. 12, 605-620.

Xu, H. M., Liang, Y., Chen, Q., Wu, Q. N., Guo, Y. M., Shen, G. P., Zhang, R. H., He, Z. W., Zeng, Y. X., Xie, F. Y., and Kang, T. B. (2011). Correlation of Skp2 overexpression to prognosis of patients with nasopharyngeal carcinoma from South China. Chin. J. Cancer 30, 204-212.

Yu, Z. K., Gervais, J. L., and Zhang, H. (1998). Human CUL-1 associates with the SKP1/SKP2 complex and regulates $\mathrm{p} 21(\mathrm{CIP} 1 / \mathrm{WAF} 1)$ and cyclin D proteins. Proc. Natl. Acad. Sci. U.S.A. 95, 11324-11329.

Zaytseva, Y. Y., Wang, X., Southard, R. C., Wallis, N. K., and Kilgore, M. W. (2008). Down-regulation of PPARgammal suppresses cell growth and induces apoptosis in MCF-7 breast cancer cells. Mol. Cancer 7, 90.

Zhang, H. Y., and Sun, H. (2010). Upregulation of Foxp3 inhibits cell proliferation, migration and invasion in epithelial ovarian cancer. Cancer Lett. 287, 91-97.

Zheng, W. Q., Zheng, J. M., Ma, R., Meng, F. F., and Ni, C. R. (2005). Relationship between levels of Skp2 and P27 in breast carcinomas and possible role of Skp2 as targeted therapy. Steroids 70, 770-774.

Zuo, T., Liu, R., Zhang, H., Chang, X., Liu, Y., Wang, L., and Zheng, P. (2007a). FOXP3 is a novel transcriptional repressor for the breast cancer oncogene SKP2. J. Clin. Invest. 117, 3765-3773.
Zuo, T., Wang, L., Morrison, C., Chang, X., Zhang, H., Li, W., Liu, Y., Wang, Y., Liu, X., Chan, M. W., Liu, J. Q., Love, R., Liu, C. G., Godfrey, V., Shen, R., Huang, T. H., Yang, T., Park, B. K., Wang, C. Y., and Zheng, P. (2007b). FOXP3 is an X-linked breast cancer suppressor gene and an important repressor of the HER-2/ErbB2 oncogene. Cell 129, 1275-1286.

Conflict of Interest Statement: The authors declare that the research was conducted in the absence of any commercial or financial relationships that could be construed as a potential conflict of interest.

Received: 10 November 2011; paper pending published: 21 November 2011; accepted: 17 December 2011; published online: 04 January 2012.

Citation: Wang Z, Fukushima $H$, Inuzuka H, Wan L, Liu P, Gao D, Sarkar FH and Wei W (2012) Skp2 is a promising therapeutic target in breast cancer. Front. Oncol. 1:57. doi: 10.3389/fonc.2011.00057

This article was submitted to Frontiers in Molecular and Cellular Oncology, a specialty of Frontiers in Oncology.

Copyright (c) 2012 Wang, Fukushima, Inuzuka, Wan, Liu, Gao, Sarkar and Wei. This is an open-access article distributed under the terms of the Creative Commons Attribution Non Commercial License, which permits noncommercial use, distribution, and reproduction in other forums, provided the original authors and source are credited. 